\title{
NEW INSIGHTS IN THE NATURAL HISTORY OF EARLY-ONSET MULTIPLE SCLEROSIS
}

\author{
Romana Homorodean', Lacramioara Perju-Dumbrava ${ }^{1,2}$ \\ ${ }^{1}$ Department of Neurology I, County Emergency Hospital of Cluj, Cluj-Napoca \\ 2"Iuliu Hatieganu” University of Medicine and Pharmacy, Cluj-Napoca
}

\begin{abstract}
Background. The early onset forms of Multiple Sclerosis (Paediatric MS, P-MS) have a low frequency, occurring in $2 \%$ to $10 \%$ of all MS cases. Age at diagnosis is a key feature for disease progression and prognosis in MS patients. Objective. To evaluate the clinical features and course of MS in patients having the disease onset before the age of 18 , according to age at diagnosis.

Methods. Patients with MS onset before age of 18 identified from Basse-Normandie MS regional registry, were included in the study. Patients were divided in two groups according to the age at MS diagnosis: the first group (group A) of patients in whom MS diagnosis was set before age of 18 and the second group (group B) including patients older than 18 years at MS diagnosis. Data related to clinical features and disease course were evaluated and compared between the two groups and also with some clinical data of adult MS patients included in the same regional database.

Results. A total of 41 patients with P-MS were included in the study, represented by 20 patients in group A and 21 patients in group B. Patients from group A had a more rapid progression to mild handicap (EDSS 3.0) than patients from group $B$ (time to reach EDSS 3.0 of 6.34 vs 14.36 years; $p=0.02$ ) and they reached this level of disability at a younger age ( 21.17 vs 29.79 years; $p=0.002)$. The duration of the first remission was significantly shorter in group $A(1.44$ vs 5.14 years; $p<0.001)$ and the evolution to secondary-progressive $(S P)$ form was faster when compared to group $B$ (14.97 vs 27.57 years; $p=0.01$ ). Patients from group A had a greater risk to reach EDSS 3.0 or EDSS 6.0 as well as a greater probability to reach SP form $(p<0.001)$ than patients from group B. Progression index $(P I)$ of the entire paediatric group was significantly lower than mean $\mathrm{PI}$ in the adult group $(0.29 \mathrm{vs} 0.45 ; p=0.003)$ but not significantly different between the two groups ( 0.37 in group A vs 0.21 in group $B)$.

Conclusion. P-MS forms show some clinical peculiarities, especially those with the age of diagnosis less than 18 years. They seem to have a more severe clinical MS course and a faster progression of disability.
\end{abstract}

Keywords: paediatric multiple sclerosis, epidemiology, course

\section{INTRODUCTION}

Multiple sclerosis (MS) is a heterogenous chronic demyelinating disease of the central nervous system (CNS) of presumed autoimmune etiology, characterized by localized areas of inflammation, demyelination, axonal loss and gliosis in the brain and spinal cord $(1,2)$. It represents the most common neurological cause of progressive disability in young adults $(3,4)$.

The clinical presentation and course of the disease are highly variable $(1,5)$. The onset of symptoms usually occurs between age of 20 to 40 but the age at onset is considerably variable (6-8). The clinical disease onset before age of 10 and after 50 years is considered relatively rare $(9,10)$. Previous studies have shown that early-onset MS (Paediatric
MS; P-MS) occurs in 2 to $10 \%$ of all MS cases and less than $1 \%$ of all MS cases have an onset earlier that age of 10 (10-12).

Although the majority of MS patients are diagnosed in early or mid-adulthood, the clinical onset of MS is being increasingly recognized in childhood due to the current diagnosis techniques (1317). However, when compared to adult-onset, P-MS cause greater diagnosis difficulties due to the age-related higher frequency of acute disseminated encephalomyelitis (ADEM) and neurometabolic white matter diseases (18-21). Moreover, distinction between ADEM and first presentation of MS is all the more difficult because it has important prognosis and therapeutic implications (22-24).

Despite a large literature, there was no certainty concerning onset symptoms, natural history or 
prognostic factors of P-MS (6,25-28). Indeed, some previous studies suggested that P-MS has a more favourable clinical course than the adult form $(29,30)$, but these data were not confirmed by more recent ones $(6,12,31)$. A more frequent polysymptomatic onset when compared to adult-MS, is also still debated $(9,11,12)$. Concerning prognostic factors they have been evaluated in different studies with inhomogeneous results (31-34). However relapse rates, especially in the early phase of the disease, the interval between the first and the second attack, the level of disability after one year, an early shift to secondary progressive (SP) form have been proposed as negative prognostic factors by some authors (6,35-38), but not confirmed by others (3941).

Nevertheless, in spite of a probable slower initial evolution, the level of disability of P-MS patients at a given age seems to be generally higher (36). In addition, recent data underline the role of age in the natural history of the disease as a very important clinical prognostic factor (39-41). Most studies on P-MS compared clinical paediatric data to adult cohorts, therefore it would be interesting to uncover additional data related to age among P-MS patients.

We conducted an epidemiological observational retrospective study on a cohort of patients from Basse-Normandie regional area, including 3 counties (1.45 M inhabitants), having their clinical onset of MS before the age of 18 years.

The objectives were to evaluate the clinical course of P-MS according to age at diagnosis, and to identify factors affecting the progression of the disease. A comparison was also made with a cohort of adult MS patients (having the disease onset after the age of 18).

\section{METHODS}

\section{Patients and inclusion criteria}

Paediatric cases were defined as having MS onset before the age of 18 , according to the newer definitions of these forms (42-45). The cases were identified within the database of the regional MS network funded by the French National Health Insurance system in 2000. This network includes all the neurologists of the regional area who fed with their cases the database according to technical and epidemiological rules.

For case selection we used the following inclusion criteria: 1) Diagnosis of MS established according to Poser or McDonald criteria (modified or not) depending of the date of diagnosis (46-50); 2) Clinical onset (first documented sign or symptom) before age of $18(42,43)$; 3) At least one neurological assessment in the late 5 years prior to the study inclusion.

Patients were classified in two groups according to the age at MS diagnosis: the first group (group A) of patients in whom MS diagnosis was set before age of 18 and the second group (group B) including patients older than 18 years at MS diagnosis.

The following characteristics were collected in patients: age at onset, age at diagnosis, type of clinical onset (first documented sign or symptom) classified into 6 categories (optic neuritis, sensory and pyramidal signs, brainstem and cerebellar signs, and gait disorders), the monosymptomatic or ploysymptomatic presentation at onset, initial form of disease (primary progressive form - PPMS or relapsing-remitting form - RRMS), relapses history (date, number), first episode of remission (duration, completeness), level of disability using the Expanded Disability Status Scale score (EDSS) (51).

Disease progression was evaluated in terms of time from the clinical onset to confirmed SP-MS conversion, time to confirmed EDSS 3.0, time to confirmed EDSS 6.0, and progression index of the disease (PI) as a measure of accumulated disability over time (PI $=$ EDSS score/disease duration in years).

The data about disease-modifying treatment (DMT) was not collected from the cohort. Evaluation of DMT drugs side-effects, causes of treatment change or stopping were not set for the study purposes.

The adult MS group consisted of the patients in the same regional database having the disease onset after age of 18. Analyzed items in this group were limited to sex ratio, age at onset and diagnosis, form of the disease, EDSS at last follow-up, and progression index.

The clinical characteristics and disease course were classified according to standardized definitions $(3,36)$. All EDSS scores in this study refer to residual neurological disability following clinical attacks. Initial EDSS represents neurological disability at the time when MS diagnosis was established.

\section{Statistical analysis}

Discrete data are presented as frequencies and percentages, continuous variables as means and standard deviations or median and quartiles. Clinical characteristics of the two paediatric patients were compared using chi-square or Fisher exact tests for qualitative variables, non-parametric Mann-Whitney tests for continuous measures. 
Time-to-event analyses (Cox proportional hazard models) were performed to identify factors affecting disease progression (time to SP-MS, time to EDSS 3.0, time to EDSS 6.0). Hazard ratios (HR) and 95\% confidence intervals (CI) were computed for each potential factor. Separate Kaplan-Meier curves were produced in the two study groups for each of the three endpoints. Time to first disease-modifying treatment was also compared between the two groups using a Cox model. Considering the small number of patients, multivariate analyses were not attempted. Statistical signification was set at $p<0.05$. Analyses were conducted using SPSS software, version 14.0.

\section{RESULTS}

A total of 43 patients with MS onset before age of 18 were identified in the database. Two patients were excluded from the study because of insufficient medical information (relapse history, EDSS scores), leaving 41 patients to be studied. The age of diagnosis was before 18 years for 20 patients (group A) and at or after 18 years for 21 patients (group B). The adult group was represented by 605 patients of the database. The clinical characteristics of the P-MS patients are described and compared between the two groups in Table 1 .

\section{Sex ratio and age}

For the whole group of P-MS patients, the overall female to male ratio was of 3.1 compared to 2.6 in the adults group. The female to male ratio did not differ significantly between the groups A and B (2.3 vs $4.3, \mathrm{p}=0.48$ ).

The mean age at MS onset was of 14.5 (vs 33.9 in the adult MS group). Only 2 of the 41 patients $(4.9 \%)$ were younger than age of 10 at onset. There is a male predominance before age of 12 in contrast to a female predominance between ages of 12 and 18. The age intervals with the highest frequency at MS onset were $14-15$ years in group A $(40.0 \%)$ and 16-17 years in group B (52.4\%). 3 patients in group $\mathrm{A}$ and none in group B were younger than 11 years at onset.

The mean age at MS diagnosis was 19.3 years in the whole paediatric group (15.3 years in group A vs 23.2 years in group B) and 37.4 years in the adult group.

\section{Symptoms, disease form and relapses}

For the entire P-MS cohort, the most frequent onset symptoms were optic neuritis $(34.1 \%)$ and sensory alterations $(31.7 \%)$ (Table 2$)$. Females presented more frequently optic neuritis (41.9\%); whereas males - pyramidal signs $(30.0 \%)$.

TABLE 1. Clinical characteristics of P-MS patients according to age at diagnosis $(n=41)$.

\begin{tabular}{|c|c|c|c|c|}
\hline Characteristics & $\begin{array}{c}\text { Group A } \\
n=20\end{array}$ & $\begin{array}{c}\text { Group B } \\
n=21\end{array}$ & $\begin{array}{c}\text { Total } \\
n=41\end{array}$ & $\mathrm{p}^{*}$ \\
\hline Female/male (ratio) & $14 / 6(2.3: 1)$ & $17 / 4(4.3: 1)$ & $31 / 10(3.1: 1)$ & 0.48 \\
\hline \multicolumn{5}{|l|}{ Age at MS onset $* *$} \\
\hline mean $(\mathrm{sd})$ & $13.6(3.7)$ & $15.2(1.5)$ & $14.5(2.9)$ & \\
\hline median (quartiles) & $15.0(14.2,17.0)$ & $16.0(14.0,16.0)$ & $15.0(14.0,16.0)$ & 0.12 \\
\hline \multicolumn{5}{|l|}{ Initial form of disease } \\
\hline RR-MS (n (\%)) & $19(95.0 \%)$ & $21(100 \%)$ & $40(97.6 \%)$ & \multirow{2}{*}{0.49} \\
\hline PP-MS (n (\%)) & $1(5.0 \%)$ & $0(0 \%)$ & $1(2.4 \%)$ & \\
\hline Follow-up duration** & & & - & \\
\hline mean $(\mathrm{sd})$ & $11.5(8.5)$ & $23.1(14.0)$ & $17.4(12.9)$ & \\
\hline median (quartiles) & $10.5(3.7,21.0)$ & $18(10.5,36.0)$ & $13.1(6.5,25.2)$ & 0.004 \\
\hline \multicolumn{5}{|l|}{ Annual relapse rate } \\
\hline mean $(\mathrm{sd})$ & $0.97(0.66)$ & $0.48(0.39)$ & $0.72(0.59)$ & \\
\hline median (quartiles) & $0.90(0.38,1.7)$ & $0.40(0.18,0.54)$ & $0.50(0.26,1.1)$ & 0.011 \\
\hline \multicolumn{5}{|c|}{ Number of relapses ( $1^{\text {st }}$ year $)$} \\
\hline mean $(\mathrm{sd})$ & $2.1(1.3)$ & $1.2(0.54)$ & $1.6(1.0)$ & \\
\hline median (quartiles) & $2.0(1.0-3.0)$ & $1.0(1.0-1.0)$ & $1.0(1.0-2.0)$ & 0.021 \\
\hline \multicolumn{5}{|c|}{ Number of relapses ( $1^{\text {st }}$ and $2^{\text {nd }}$ year $)$} \\
\hline mean $(s d)$ & $3.1(2.3)$ & $1.7(1.4)$ & $2.4(2.0)$ & \\
\hline median (quartiles) & $2.0(1.0-5.0)$ & $1.0(1.0-2.0)$ & $1.0(1.0,4.0)$ & 0.030 \\
\hline \multicolumn{5}{|c|}{ Duration of first remission** } \\
\hline mean $(\mathrm{sd})$ & $1.5(2.7)$ & $5.0(4.4)$ & $3.3(4.0)$ & \\
\hline median (quartiles) & $0.42(0.17-2.0)$ & $4.0(1.3-8.0)$ & $1.8(0.42,4.8)$ & $<0.001$ \\
\hline
\end{tabular}

* $\mathrm{p}$ value refers to the comparison between group $\mathrm{A}$ and $\mathrm{B}$;

** Values are expressed as years 
TABLE 2. Distribution of P-MS patients according to sex and initial symptoms $(n=41)$.

\begin{tabular}{|c|c|c|c|c|c|c|c|}
\hline \multirow[b]{2}{*}{ Initial symptoms } & \multicolumn{3}{|c|}{$\begin{array}{c}\text { Group A } \\
\text { MS diagnosis before age of } 18\end{array}$} & \multicolumn{3}{|c|}{$\begin{array}{c}\text { Group B } \\
\text { MS diagnosis after age of } 18\end{array}$} & \multirow[b]{2}{*}{$p^{*}$} \\
\hline & $\begin{array}{c}\text { Males } \\
\mathrm{n}=6 \\
\%\end{array}$ & $\begin{array}{c}\text { Females } \\
\mathrm{n}=14 \\
\%\end{array}$ & $\begin{array}{c}\text { Total } \\
\mathrm{n}=20 \\
\%\end{array}$ & $\begin{array}{c}\text { Males } \\
\mathrm{n}=4 \\
\% \\
\end{array}$ & $\begin{array}{c}\text { Females } \\
\mathrm{n}=17 \\
\%\end{array}$ & $\begin{array}{c}\text { Total } \\
\mathrm{n}=21 \\
\%\end{array}$ & \\
\hline Optic neuritis & 16.7 & 42.9 & 35 & 0 & 41.2 & 33.3 & 1 \\
\hline Sensory signs & 16.7 & 42.9 & 35 & 25 & 29.4 & 28.6 & 0.75 \\
\hline Pyramidal signs & 16.7 & 21.4 & 20 & 50 & 17.6 & 23.8 & 1 \\
\hline Brainstem signs & 33.3 & 14.3 & 20 & 0 & 29.4 & 23.8 & 1 \\
\hline Cerebellar signs & 16.7 & 21.4 & 20 & 25 & 5.9 & 9.5 & 0.41 \\
\hline Gait disorders & 0 & 21.4 & 15 & 0 & 5.9 & 4.8 & 0.34 \\
\hline Polysymptomatic onset & 16.7 & 50.0 & 40 & 0 & 23.5 & 19 & 0.18 \\
\hline
\end{tabular}

* $p$ value refers to the comparison of the "Total" columns in group $A$ and $B$

In the two paediatric groups, only one patient (group A) had initially PPMS course, the other 40 cases $(97.6 \%)$ being categorized as RRMS. In the adult group, the RRMS initial course occurred in $84.7 \%$ of cases and the PPMS in $15.3 \%$ (data available for 594 patients). The difference was not significant $(\mathrm{p}=0.08)$.

Of the overall paediatric MS patients with initially RRMS, only 8 (19.5\%) had less than 5 relapses during their disease course. The annual relapse rate (on the whole course of the disease) was significantly lower in group B $(\mathrm{p}=0.011$, Table 1$)$. The number of relapses was also significantly lower in group $B$ during the first year $(p=0.021)$ and the first two years of the disease $(p=0.03)$. Of the 19 patients from group A with initially RRMS, 11 patients $(57.9 \%)$ had at least one additional relapse during the first year and 13 (68.4\%) during the first two years following MS onset. In group B only 4 $(19.0 \%)$ had at least one additional relapse during the first year and $6(28.6 \%)$ during the first two years following MS onset.

The delay between the first two relapses was largely longer in group B than in group A (mean 5.14 vs 1.44 years respectively, $\mathrm{p}<0.001) .13$ patients $(68.4 \%)$ in group A and $19(90.5 \%)$ in group $\mathrm{B}$ had a complete recovery after the first attack $(\mathrm{p}=0.12)$.

\section{Clinical course, disability and progression}

The overall median follow-up (MS onset to current study inclusion) was of 13.1 years, but the follow-up period was significantly longer in group B compared to group A $(p=0.004$, Table 1$)$. In addition, $65.9 \%$ of all patients had been followed for more than 10 years, representing $50.0 \%$ of group A and $81.0 \%$ of group B.

From 19 subjects of group A having initially RRMS, 5 (26.3\%) developed SPMS in the whole period of follow-up, after a median time of 17 years. In group B, 7 subjects out of 21 (33.33\%) evolved to SPMS, after a median time of 27 years. The factors significantly affecting the conversion to SPMS were represented by the age at diagnosis below $18(p=0.007)$, the presence of brainstem and cerebellar signs at onset $(p=0.05$ and $p=0.02$ respectively) and the annual relapse rate, with a doubling of the probability to reach SPMS per $10 \%$ increase of the rate $(p=0.01)$.

Kaplan-Meier survival curves according to age at diagnosis are presented in Fig. 1. The 50\% risk for the switch from RRMS to SPMS (median survival time) is reached 18.8 years after onset in group A compared to 32.0 years in group B.

The overall median EDSS at last follow-up was 3.5 (quartiles: $1.5,6.5$ ) in the P-MS cohort, compared to 3.5 (quartiles: $1.5,6.0$ ) in the adult group (data available for 533 adult patients). A median value of 3.25 (quartiles: 1.1, 5.75) was observed in group A versus 3.5 (quartiles: 2.25, 6.5) in group B $(p=0.47)$. Median EDSS at the time of MS diagnosis was 2.0 (quartiles: 1.0, 2.0) in group A compared to 2.0 (quartiles: 1.0, 2.75) in group B $(\mathrm{p}=0.87)$.

EDSS score of 3.0 at last follow-up had been reached by 11 patients in group A after a median time of 7 years, and 13 patients in group B after a median time of 13 years. Factors significantly affecting progression to EDSS $\geq 3.0$ were represented by the age at diagnosis below $18(\mathrm{p}=0.014)$, the presence of cerebellar signs at onset $(p=0.005)$, the polysymptomatic onset $(p=0.03)$ and the annual relapse rate $(p<0.001)$. A negative association with the duration of the 1 st remission was close to significance, with a $9 \%$ decrease of the probability to progress to EDSS $\geq 3.0$ per 1-year increase of the duration $(p=0.066)$. Kaplan-Meier survival curves according to age at diagnosis are presented in Fig. 2. 

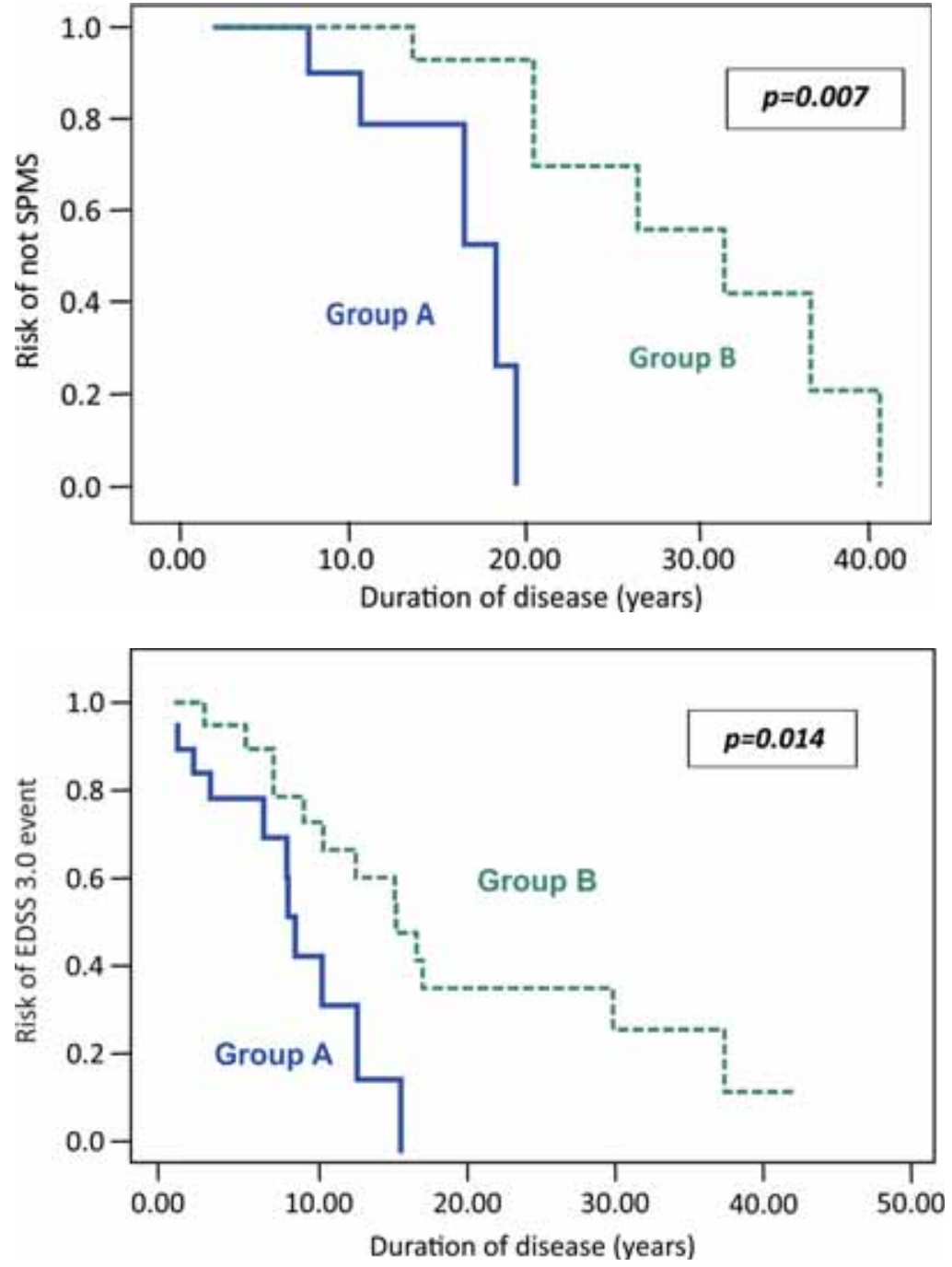

FIGURE 1. Kaplan-Meier survival curves of the probability of not reaching secondary progression (SPMS) in groups $A$ and $B$.
FIGURE 2. Kaplan-Meier survival curves of the probability of not reaching EDSS score of 3.0 in groups $A$ and $B$.
The median survival time is 8.5 years in group A compared to 15.4 years in group B.

The EDSS score of 6.0 at last follow-up had been reached by 5 patients in group A after a median time of 15 years, and 9 in group B after a median time of 24 years. Factors significantly affecting progression to EDSS $\geq 6.0$ were represented by the age at diagnosis under $18(\mathrm{p}=0.016)$, the presence of cerebellar signs at onset $(p=0.043)$ and a higher annual relapse rate $(p=0.006)$. Association with brainstem signs at onset was close to significance $(p=0.057)$. KaplanMeier survival curves according to age at diagnosis are presented in Fig. 3. The median survival time is 15.0 years in group A compared to 29.3 years in group B.

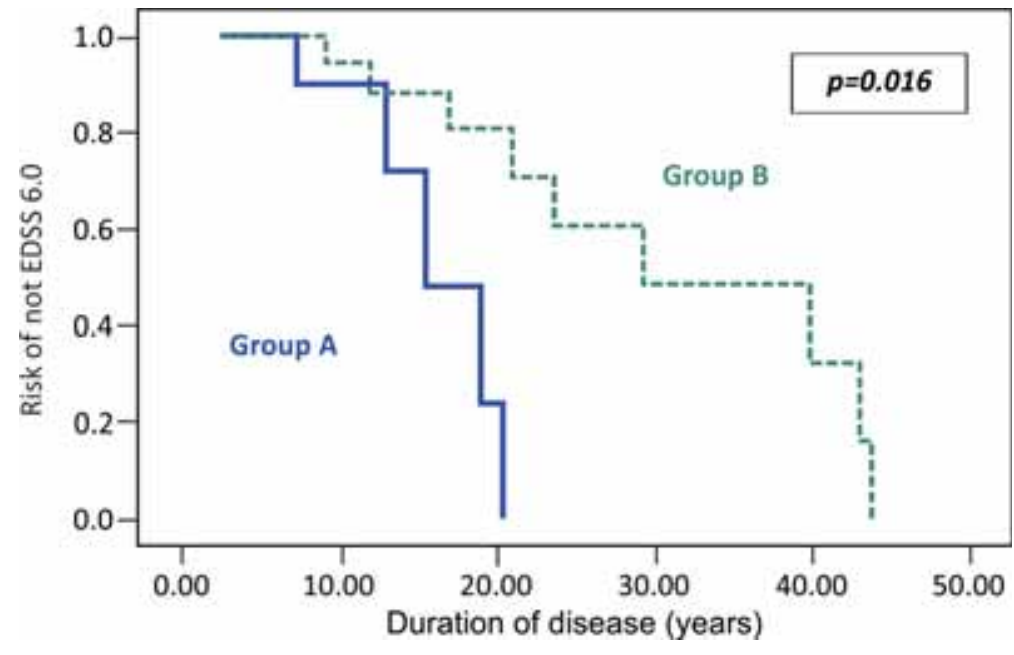

FIGURE 3. Kaplan-Meier survival curves of the probability of not reaching EDSS score of 6.0 in groups $A$ and $B$. 
To better evaluate MS accumulated disability over time, we calculated the progression index (PI) in the two study groups. The patients in group A had a median PI of 0.30 (quartiles: 0.11, 0.49) compared to 0.18 (quartiles: $0.10,0.24)$ in group $B(p=$ 0.09 ). The progression index was however significantly lower in all P-MS patients (median: 0.23, quartiles: $0.11,0.41)$ than in the adult MS group (median: 0.30 , quartiles: $0.17,0.52, \mathrm{p}=0.036$, data available for 533 adult MS patients).

\section{DISCUSSION}

In agreement with previous studies $(6-8,36)$, our study shows a female preponderance among P-MS without significant difference when compared to adults. The distribution by age and sex confirms a female preponderance after the age of 13 , whereas most patients are male before the age of 12 (female/ male ratio $<1)$. In addition, all patients younger than 12 years at MS onset are males. The highest female to male ratio (5.4:1) was observed in the pubertal and postpubertal period (14-17 years). Thus, as in other studies, our data support the theory of a role of sexual hormonal influence on MS onset $(6,36,37)$.

The overall most common initial signs are optic neuritis $(34.1 \%)$ and sensory signs $(31.7 \%)$ consistent with the study of Boiko and colab. (6), even if the observed frequency of this symptoms is higher in our study with a slightly predominance of optic neuritis. Girls have most often optic neuritis whereas boys have a predominance of motor symptoms at disease onset. Contrary to Boiko data (6), in our study no boy presents gait disorder at clinical onset. According to other studies, onset symptoms of brainstem or cerebellum involvement and a high rate of relapses were associated with a more frequent transformation to secondary progressive MS $(36,38)$.

PPMS form has been rarely reported in P-MS patients in the most previous studies $(6-8,36)$. Our study confirmed that the initial clinical course was mainly relapsing-remitting and that frequency of progressive form is largely lower in P-MS compared to adult MS patients (2.4\% vs $15.3 \%$ ).

Concerning the early phase of the disease, there is a possible relationship between the duration of the first remission and the time to reach EDSS 3.0. In addition, the time to reach EDSS 6.0 is negatively associated to the frequency of relapses.

At last follow-up, the level of neurological disability was comparable between paediatric MS patients and adult patients (median EDSS score of 3.5 in the 2 groups). In agreement with several previous studies $(6,27,31,36)$ the rate of disease progression was slower in P-MS patients compared to adult patients. Nevertheless, these patients reach disability landmarks at a younger age that is clearly lower than the mean age at MS onset or diagnosis of our adult cohort. This was in agreement with recent papers which emphasise the role of age in the natural history of MS $(7,8,39,40)$.

One surprising result from our cohort analysis deserves some specific comments. Indeed, followup period of group A is clearly lower than in group B despite a clearly worst clinical evolution (significant shorter time to reach EDSS 3.0 or 6.0 and shift to SP-MS form). The more immediate and obvious explanation will be that MS diagnosis in paediatric patients was difficult (even not done) in the older period for our cohort and more easily and frequently done in a more recent period. This could be explained by the use in the last 20 years of validated diagnosis criteria and the parallel improvement of diagnostic methods. This was confirmed by looking individually to the year of diagnosis for each patient of our cohort showing that no patient had MS diagnosis before age of 18 before 1980 but all after that, with $50 \%$ having this diagnosis since 2000.

\section{CONCLUSION}

Among P-MS patients, those getting MS diagnosis before age of 18 (group A) have a more rapid progression to mild or significant disability (EDSS 3.0 or 6.0) and reach EDSS 3.0 at a younger age. The duration of first remission is significantly shorter in this group of patients getting MS diagnosis before age of 18 and the number of relapses during the first two years is also significantly higher as well as the annual relapse rate. In addition, these patients have a faster evolution to secondary progressive MS despite a PI that was not significantly different from the group B of P-MS patients.

In summary, $\mathrm{P}-\mathrm{MS}$ forms have clinical features and disease course different from that we classically observed in adult patients, especially those who had confirmed MS diagnosis before the age of 18 years. This raises the question of early use of disease-modifying treatment owing to the particular clinical course of these patients. Although this study adds some important elements to our knowledge on P-MS, further studies with greater cohorts of patients are needed to fully delineate the clinical features and course of these MS and their response to early therapy. 


\section{Acknowledgements}

The authors appreciate the help of Prof. Defer Gilles, Cean, France in patient recruitment, study development and also manuscript preparation. We are grateful to all those who were involved in the process of data collection or statistics.

\section{REFERENCES}

1. Milo R., Miller A. Revised diagnostic criteria of multiple sclerosis. Autoimmun Rev. 2014; 13(4-5):518-24.

2. Gajofatto A., Calabrese M., Benedetti M.D., et al. Clinical, MRI, and CSF markers of disability progression in multiple sclerosis. Dis Markers. 2013; 35(6):687-99.

3. Noseworthy J.H., Lucchinetti C., Rodriguez M., et al. Multiple sclerosis. N Engl J Med. 2000; 343(13):938-52.

4. Compston A., Coles A. Multiple sclerosis. Lancet. 2008; 372(9648):1502-17.

5. Renoux C. Natural history of multiple sclerosis: long-term prognostic factors. Neurol Clin. 2011; 29(2):293-308.

6. Boiko A., Vorobeychik G., Paty D., et al. Early onset multiple sclerosis: a longitudinal study. Neurology. 2002; 59(7):1006-10.

7. Confavreux C., Vukusic S. The clinical course of multiple sclerosis. Handb Clin Neurol. 2014; 122:343-69.

8. Vukusic S., Confavreux C. Natural history of multiple sclerosis. Presse Med. 2010; 39(3):359-62.

9. Simone I.L., Carrara D., Tortorella C., et al. Course and prognosis in early-onset MS: comparison with adult-onset forms. Neurology. 2002; 59(12):1922-8.

10. Harding K.E., Liang K., Cossburn M.D., et al. Long-term outcome of paediatric-onset multiple sclerosis: a population-based study. J Neurol Neurosurg Psychiatry. 2013; 84(2):141-7.

11. Chou I.J., Wang H.S., Whitehouse W.P., et al. Paediatric Multiple Sclerosis: Update on Diagnostic Criteria, Imaging, Histopathology and Treatment Choices. Curr Neurol Neurosci Rep. 2016; 16(7):68.

12. Gadoth N. Multiple sclerosis in children. Brain Dev. 2003; 25(4):229-32.

13. Pohl D., Rostasy K., Reiber H., et al. CSF characteristics in early-onset multiple sclerosis. Neurology. 2004; 63(10):1966-8.

14. Ruggieri M., lannetti P., Polizzi A., et al. Multiple sclerosis in children under 10 years of age. Neurol Sci. 2004; 25 Suppl 4:S326-35.

15. Callen D.J., Shroff M.M., Branson H.M., et al. MRI in the diagnosis of pediatric multiple sclerosis. Neurology. 2009; 72(11):961-7.

16. Rubin J.P., Kuntz N.L. Diagnostic criteria for pediatric multiple sclerosis. Curr Neurol Neurosci Rep. 2013; 13(6):354

17. Sedani S., Lim M.J., Hemingway C., et al. Paediatric multiple sclerosis: examining utility of the McDonald 2010 criteria. Mult Scler. 2012; 18(5):679-82.

18. Hahn J.S., Pohl D., Rensel M., et al. Differential diagnosis and evaluation in pediatric multiple sclerosis. Neurology. 2007; 68(suppl 2):S13-22.

19. Narula S., Banwell B. Pediatric Demyelination. Continuum (Minneap Minn). 2016; 22(3, Multiple Sclerosis and Other Demyelinating Diseases):897-915.

20. Dale R.C., de Sousa C., Chong W.K., et al. Acute disseminated encephalomyelitis, multiphasic disseminated encephalomyelitis and multiple sclerosis in children. Brain. 2000; 123 Pt 12:2407-22.

21. Brass S.D., Caramanos Z., Santos C., et al. Multiple sclerosis vs acute disseminated encephalomyelitis in childhood. Pediatr Neurol. 2003; 29(3):227-31.

22. Pohl D., Hennemuth I., von Kries R., et al. Paediatric multiple sclerosis and acute disseminated encephalomyelitis in Germany: results of a nationwide survey. Eur J Pediatr. 2007; 166(5):405-12.

23. Hynson J.L., Kornberg A.J., Coleman L.T., et al. Clinical and neuroradiologic features of acute disseminated encephalomyelitis in children. Neurology. 2001; 56(10):1308-12.

24. Tardieu M., Mikaeloff $Y$. What is acute disseminated encephalomyelitis (ADEM)? Eur J Paediatr Neurol. 2004; 8(5):239-42.

25. Tenembaum S.N. Acute disseminated encephalomyelitis. Handb Clin Neurol. 2013; 112:1253-62.

26. Ghezzi A., Ruggieri M., Trojano M., et al. Italian studies on early-onset multiple sclerosis: the present and the future. Neurol Sci. 2004; 25 Suppl 4:S346-9.

27. Tardieu M., Mikaeloff Y. Multiple sclerosis in children. Int MS J. 2004; 11(2):36-42.

28. Renoux C., Vukusic S., Mikaeloff Y., et al. Natural history of multiple sclerosis with childhood onset. N Engl J Med. 2007; 356(25):2603-13.

29. Duquette P., Murray T.J., Pleines J., et al. Multiple sclerosis in childhood: clinical profile in 125 patients. J Pediatr. 1987; 111(3):359-63.

30. Mezzapesa D.M., Rocca M.A., Falini A., et al. A preliminary diffusion tensor and magnetization transfer magnetic resonance imaging study of early-onset multiple sclerosis. Arch Neurol. 2004; 61(3):366-8.

31. Deryck O., Ketelaer P., Dubois B. Clinical characteristics and long term prognosis in early onset multiple sclerosis. J Neurol. 2006; 253(6):720-3.

32. Ness J.M., Chabas D., Sadovnick A.D., et al. Clinical features of children and adolescents with multiple sclerosis. Neurology. 2007; 68(16 Suppl 2):S37-S45.

33. Mikaeloff Y., Caridade G., Assi S., et al. Prognostic factors for early severity in a childhood multiple sclerosis cohort. Pediatrics. 2006; 118(3):1133-9.

34. Degenhardt A., Ramagopalan S.V., Scalfari A., et al. Clinical prognostic factors in multiple sclerosis: a natural history review. Nat Rev Neurol. 2009; 5(12):672-82.

35. Mowry E.M. Natural history of multiple sclerosis: early prognostic factors. Neurol Clin. 2011; 29(2):279-92.

36. Compston A., Confavreux C., Lassman H., et al. McAlpine's multiple sclerosis. 4th edition. Philadelphia: Churchill Livingstone Elsevier; 2006.

37. Ghezzi A., Pozzilli C., Liguori M., et al. Prospective study of multiple sclerosis with early onset. Mult Scler. 2002; 8(2):115-8.

38. Ghezzi A. Clinical characteristics of multiple sclerosis with early onset. Neurol Sci. 2004; 25 Suppl 4:S336-9.

39. Confavreux C., Vukusic S. Natural history of multiple sclerosis: a unifying concept. Brain. 2006; 129(Pt 3):606-16.

40. Confavreux C., Vukusic S. Age at disability milestones in multiple sclerosis. Brain. 2006; 129(Pt 3):595-605.

41. Renoux $C$. Natural history of multiple sclerosis: long-term prognostic factors. Neurol Clin. 2011; 29(2):293-308.

42. Krupp L.B., Banwell B., Tenembaum S., et al. Consensus definitions proposed for pediatric multiple sclerosis and related disorders. Neurology. 2007; 68(suppl 2):S7-12.

43. Krupp L.B., Tardieu M., Amato M.P., et al. International Pediatric Multiple Sclerosis Study Group criteria for pediatric multiple sclerosis and immune-mediated central nervous system demyelinating disorders: revisions to the 2007 definitions. Mult Scler. 2013; 19(10):1261-67.

44. Chou I.J., Whitehouse W.P., Wang H.S., et al. Diagnostic modalities in multiple sclerosis: perspectives in children. Biomed J. 2014; 37(2):50-9.

45. Rubin J.P., Kuntz N.L. Diagnostic criteria for pediatric multiple sclerosis. Curr Neurol Neurosci Rep. 2013; 13(6):354.

46. Poser C.M., Paty D.W., Scheinberg L., et al. New diagnosis criteria for multiple sclerosis: guidelines for research protocols. Ann Neurol. 1983; 13(3):227-31. 
47. McDonald W.I., Compston A., Edan G., et al. Recommended diagnosis criteria for multiple sclerosis: guidelines from the international panel on the diagnosis of multiple sclerosis. Ann Neurol. 2001; 50(1):121-7.

48. Polman C.H., Reingold S.C., Edan G., et al. Diagnostic criteria for multiple sclerosis: 2005 Revisions to the "McDonald Criteria". Ann Neurol. 2005; 58(6):840-6.

49. Polman C.H., Reingold S.C., Banwell B., et al. Diagnostic criteria for multiple sclerosis: 2010 revisions to the McDonald criteria. Ann Neurol. 2011; 69(2):292-302.
50. Sedani S., Lim M.J., Hemingway C. Paediatric multiple sclerosis: examining utility of the McDonald 2010 criteria. Mult Scler. 2012; 18(5):679-82.

51. Kurtzke J.K. Rating neurological impairment in multiple sclerosis: an expanded disability status scale (EDSS). Neurology. 1983; 33(11):1444-52. 